\title{
DIRECT MEASUREMENT OF THE CCD WITH A SUBPIXEL RESOLUTION BY USING A NEW TECHNIQUE
}

\author{
K. YOSHITA, S. KITAMOTO, E. MIYATA AND H. TSUNEMI \\ Graduate School of Science, Osaka University \\ 1-1 Machikaneyama-cho, Toyonaka, Osaka 560, Japan \\ AND \\ K. C. GENDREAU \\ Goddard Space Flight Center
}

\section{Introduction}

The response function of the front-illuminated CCD, like SIS on ASCA, can be devided into three parts: the gate structure transmission, the absorption efficiency in the depletion region and the charge spreading after the photoabsorption. These effect depend on both the incident X-ray energy and the landing posion of the X-ray inside the CCD pixel. Then, the measurement of the $\mathrm{X}$-ray efficiency within the pixel is important for the response function of the CCD. By using a new technique, we performed an experiment using the SIS CCD chip in the GSFC in order to measure how the various types of events are formed.

\section{Experimental Setup}

We placed a copper mesh in front of the CCD to restrict the landing position of the X-ray inside the CCD pixel. The CCD we used has $420 \times 420$ pixels of $27 \mu \mathrm{m}$ square. The mesh has $925 \times 925$ holes at $27 \mu \mathrm{m}$ intervals. The holes are circular and about $4 \mu \mathrm{m}$ in diameter. The X-ray beam line we used is a $40 \mathrm{~m}$ long tube with an X-ray generator in the GSFC.

The alignment between the mesh and the CCD is slightly titled, which creates a moire pattern. Refering to the moire pattern, we can identify the mutual alignment between the mesh and the CCD. Then, we can determine the input position of the X-rays within the pixel. 


\section{Data Analysis}

If we know the input position of the X-rays within the pixels, we can reconstruct the pixel image. We reconstructed the pixel image for single pixel events which are formed when the X-ray is photo-absorbed well inside the pixel. We drew the image of $3 \times 3$ pixels as shown in the Figure 1 to see the pixel boundary clearly. The dark regions in it show the regions where there are very few single pixel events. These correspond to the pixel boundaries.

We also reconstructed for vertically split events, horizontally split events, $\mathrm{L} / \mathrm{square}$ events which are formed when the incident $\mathrm{X}$-ray is photo-absorbed near the pixel boundary. Figure 1, 2, 3 and 4 is the reconstructed image of Y-L X-ray $(1.9 \mathrm{keV})$ data. We can clearly see how the various types of events are formed by the landing position inside the pixel.

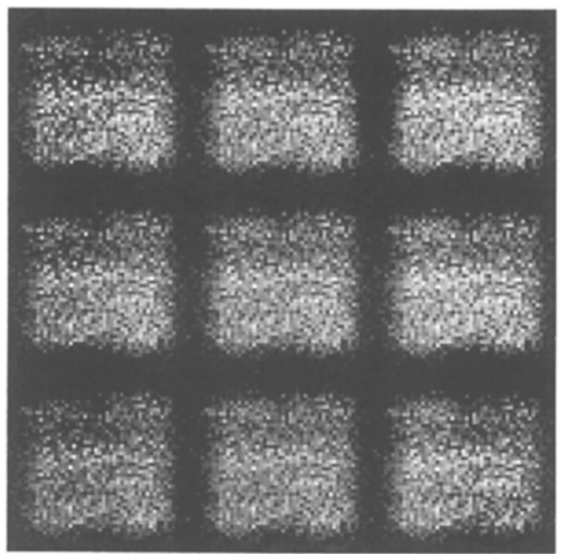

Figure 1. single pixel events

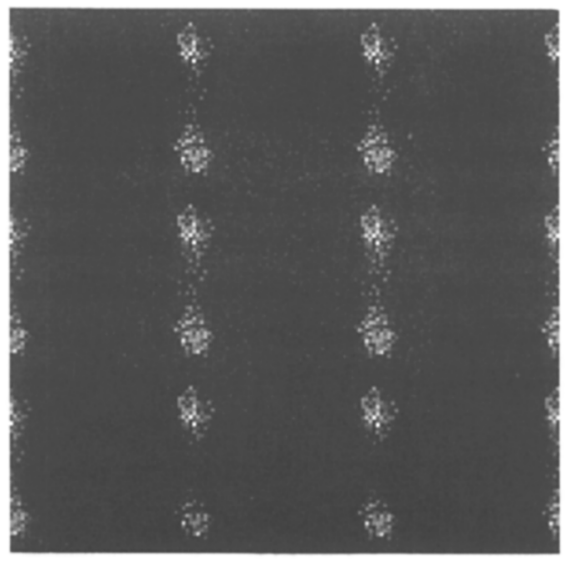

Figure 3. horizontally split events

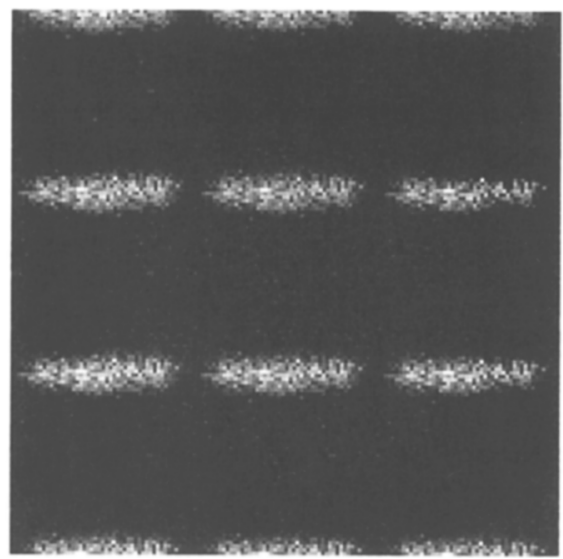

Figure 2. vertically split events

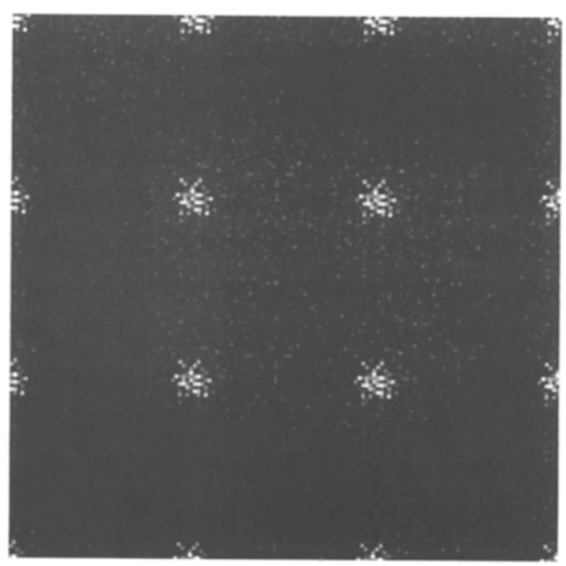

Figure 4. L/square events 